\title{
CdS, CdSe and PbS Nanostructured Thin Films Synthesized by Chemical Methods for Photovoltaic Applications
}

\author{
Yuri Vorobiev ${ }^{1}$, Francisco Willars-Rodriguez ${ }^{1}$, Fernando Chimal-Moreno $^{1}$, Rafael Ramírez-Bon$^{1}$, \\ Pavel Vorobiev ${ }^{2}$ \\ ${ }^{1}$ CINVESTAV-QuerétaroLibramiento Norponiente \#2000, Querétaro 76230, Qro, México \\ vorobiev@cinvestav.mx; fwillars@cinvestav.mx; fernando.chimal@cinvestav.mx; rrbon@cinvestav.mx \\ ${ }^{2}$ CIMAV-Monterrey, Alianza Norte 202, Parque de Investigación e Innovación Tecnológica, Apodaca 66628, N. L., \\ México, \\ pavel.vorobiev@cimav.edu.mx
}

\begin{abstract}
The chalcogenide semiconductors, like $\mathrm{CdS}, \mathrm{CdSe}, \mathrm{CdTe}, \mathrm{PbS}, \mathrm{PbSe}$ are of great interest for photovoltaic applications, in particular, due to the possibility to be part of multi-layered (tandem) photovoltaic converters. These materials are not expensive and can be produced by economic and ecologically friendly techniques like CBD (Chemical Bath Deposition) and its recent versions (SILAR Successive Ionic Layer Adsorption and Reaction, and LACBD - Light Assisted Chemical Bath Deposition). Here we study the effects of nano-porosity that is an essential feature of these techniques; the corresponding quantum confinement affects the band gap value that can be used for its monitoring to optimize the device efficiency. In general, we conclude that the quantum confinement effects caused by nano-structuration of semiconductor thin films for photovoltaic converters improve the converter's parameters.
\end{abstract}

Keywords: Chalcogenide Semiconductors, Chemical Bath Deposition, Porosity, Nano Structuration, Quantum effects.

\section{Introduction}

Today we observe continuous increase of the utilization of renewable sources of energy worldwide due to the permanent problems with fossil fuels and the ecological problems; this situation is not alien to Mexico. In particular, because of that the interest towards photovoltaic materials increases: semiconductors like gallium arsenide (GaAs - $1.43 \mathrm{eV}$ direct band gap) and cadmium telluride (CdTe - $1.5 \mathrm{eV}$ direct band gap) are ones of the most promising as light absorbers in solar cells according to Shockley and Quiesser estimations. CdSe with the band gap of $1.7 \mathrm{eV}$ that can be either n-type or p-type, and is also very promising in these applications. The common $\mathrm{CdS} / \mathrm{CdTe}$ solar cell is a simple heterojunction structure containing a p-doped CdTe light absorbing layer (can be substituted by p-CdSe film) matched with an n-doped CdS film, which acts also as a window layer. Another useful application of these or similar structure is photovoltaic light detector (sensor); for these devices Schottky diode structure might be preferable.

The other chalcogenide semiconductors (like $\mathrm{PbS}, \mathrm{PbSe}, \mathrm{PbTe}$ ) are also very interesting for photovoltaic applications: in some of them the multi exciton generation was observed, besides these can be part of efficient multi-layered light-toelectricity converters (an example of $\mathrm{CdS} / \mathrm{PbS}$ solar energy converter we presented in [1]). These materials are not expensive contrary to III-V compounds frequently used in photovoltaic devices, the techniques of their production are economic and ecologically friendly like ammonium free CBD (Chemical Bath Deposition) and its recently developed versions. Materials produced by these methods are usually nano porous; this porosity causes the corresponding quantum confinement that affects the band gap value. The corresponding band gap variation can be regulated by experimental conditions; for illustration, in $\mathrm{PbS}$ we observed the band gap variation between 0.4 and $0.8 \mathrm{eV}$ [1]. Thus, within the family of materials discussed (CdS, $\mathrm{CdSe}$ and $\mathrm{PbS}$ ) we have a set of band gaps of approximately 2.4, 1.7 and $0.8 \mathrm{eV}$ sufficient to cover the whole solar spectrum in corresponding multi-union solar cell.

This paper presents a description of preparation and investigation of semiconductor thin films mentioned above, and the discussion of the effects of quantum confinement caused by porosity on the materials optical properties. 


\section{Materials And Methods}

To improve the electrical properties of CdS films, we doped them with Sn, Al and Eu with the idea that these atoms have more valence electrons than $\mathrm{Cd}$ and thus must act as donors. Besides, Eu atoms having incomplete internal electron shell can also possess luminescent and magnetic properties that might add new options of possible material applications. The thin films of cadmium sulfide were made using the technique of ammonia free chemical bath deposition CBD assisted by ultraviolet light illumination (LACBD). As substrates we used ITO-on-glass layers, commercial glass slides and p-type silicon wafers (these in attempt to create photo diode structure). The deposition was made in a total bath volume of $50 \mathrm{ml}$ adding sequentially the following reagents: $15 \mathrm{ml}$ of $0.05 \mathrm{M}$ cadmium chloride $\left(\mathrm{CdCl}_{2} \mathrm{~J}\right.$.T.Baker ${ }^{\circledR} 99.3 \%$ purity $), 15 \mathrm{ml}$ of $0.5 \mathrm{M}$ sodium citrate $\left(\mathrm{C}_{6} \mathrm{H}_{5} \mathrm{O}_{7} \mathrm{Na}_{3}\right.$, J.T.Baker ${ }^{\circledR} 99.1 \%$ purity), $2.5 \mathrm{ml}$ of $0.5 \mathrm{M}$ potassium hydroxide (KOH, Sigma-Aldrich ( $89.13 \%$ purity), $2.5 \mathrm{ml}$ of $\mathrm{pH} 10$ buffer solution (J.T.Baker ${ }^{\circledR} 100 \%$ purity), $3.75 \mathrm{ml}$ of $0.5 \mathrm{M}$ thiourea $\left(\mathrm{CS}\left(\mathrm{NH}_{2}\right)_{2}\right.$ J.T.Baker (B) 99.6\% purity). For $\mathrm{Sn}$ doping, various concentration of $5 \mathrm{mM}$ of stannous chloride were added $\left(\mathrm{SnCl}_{2} \cdot 2 \mathrm{H}_{2} \mathrm{O}, \mathrm{J} . \mathrm{T}\right.$.Baker ( $99.3 \%$ purity); for $\mathrm{Al}$ and Eu doping, the reagents used were aluminum chloride $\left(\mathrm{AlCl}_{3} \cdot 6 \mathrm{H}_{2} \mathrm{O}\right.$, J.T.Baker ${ }^{\circledR} 99.1 \%$ purity) and europium nitrate $\left(\mathrm{Eu}\left(\mathrm{NO}_{3}\right)_{3} \cdot 5 \mathrm{H}_{2} \mathrm{O}\right.$, Sigma Aldrich ${ }^{\circledR} 98.1 \%$ purity). The solution was diluted with deionized water to have a total volume of $50 \mathrm{ml}$.

$\mathrm{CdSe}$ was prepared in a similar way using $\mathrm{Na}_{2} \mathrm{SeSO}_{3}$ instead of thiuorea that was a source of sulfur in $\mathrm{CdS}$ preparation, the complete procedure is described in [2]. The amount of sodium citrate was varied between 2.5 and $7.5 \mathrm{ml}$ in solution, for studying its effect upon the films adhesion. The best results correspond to $7.5 \mathrm{ml}$. The deposition of $\mathrm{PbS}$ film by chemical bath was presented in [1].

X-ray diffraction (XRD) of samples was measured by a multipurpose X-ray diffraction system (XRD, Rigaku Co., UltiIV, Japan) with $\mathrm{Cu}\left(\mathrm{K}_{\alpha 1 / \alpha 2}=1.5406 \AA / 1.5444 \AA\right)$ source. The diffractograms were recorded at room temperature between angles $20^{\circ}$ to $80^{\circ}$ with a scan rate of $1 \%$ min and step size $0.005^{\circ}$. Optical properties were studied in a Spectrometer (SCI Co., FilmTek 3000, CA., USA) in the wavelength range of $240 \mathrm{~nm}$ to $950 \mathrm{~nm}$; the information obtained from the measurement was used to calculate the bandgap for each sample. The work function was measured with the Kelvin probe system ((SKP) KP Technology, SKP5050), work function resolution 5-10 meV (50 $\mu \mathrm{m}$ tip), the SKP instrument is equipped with a tungstenhalogen light source to measure surface photovoltage as a function of intensity. The X-ray Photoelectron Spectroscopy (XPS) was studied using equipment XPS Escolab 250Xi that gives the chemical composition of the films and atomic binding energies. For studying the morphological properties, a Field Emission Scanning Electron Microscope was used (JEOL Ltd, JSM-7610F, Japan). The film composition was determined using energy dispersive X-ray analysis (Electron Dispersion Spectroscopy with SEM, Philips Co., XL30 ESEM, Netherland).

The measurement's errors are defined, first of all, by the corresponding device calibration. In case of XRD data, the calibration with monocrystalline silicon was performed giving the experimental error in finding the crystal lattice constants in $0.001 \AA$; that enables determination of the lattice distortion caused by substitution of the lattice atoms by another atoms different in atomic size. In optical measurements, the SCI FilmTek 3000 instrument was calibrated with 2 standards (Si wafer and $\mathrm{Si} / \mathrm{SiO}_{2}$ wafer) and the errors in measurements of transmission and reflection were less than 5\%. The accuracy in determination of samples band gap by a standard extrapolation technique we estimate as $0.01 \mathrm{eV}$. In estimation of the grain size from SEM images, the error is determined by actual dispersion of sizes: here 40 measurements were made for each sample, and then the average and standard deviation were calculated (shown in corresponding figure).

\section{Results and Discussion}

\subsection{Morphology and Structure}

The results of the morphologic study on CdS:M thin films (M being the doping metal atom) are shown in Fig. 1, here a typical morphology for a CdS films obtained from CBD technique was observed [3]. The superficial morphology exhibits a homogenous composition, absence of pin-holes, lack of cracks through the film; further the films present spherically shaped grains. It is observed that the grain size increases as a function of the dopant added to the CBD solution. SEM image of CdSe film is presented in Fig. 2. Here, same as in $\mathrm{CdS}$ case and $\mathrm{PbS}$, close-packed spherical grains are observed, with pores among them. 
The crystallographic properties of CdS thin films doped with different metals were evaluated via X-ray diffraction, the patterns are shown in Fig. 3, where two diffraction peaks are observed at $\sim 26.5^{\circ}$ and $\sim 48^{\circ}$ related with the (002) and (103) crystalline planes for the hexagonal phase of $\mathrm{CdS}$ (this behavior is observed in aluminum- and tin-doped cadmium sulfide films and undoped CdS), this information is confirmed from ICDD card No. 41-1049. Eu-doped cadmium sulfide diffractogram exhibit several peaks at $\sim 26.1^{\circ}, 36.1^{\circ}, 44.2^{\circ}, 48.4^{\circ}$ and $52.2^{\circ}$ corresponding to the planes (002), (102), (110), (103) and (112) for the hexagonal phase of CdS or Greenockite (PDF No. 41-1049). Obtained from diffractograms crystallite size was around $40 \mathrm{~nm}$ for all films except Eu-doped ones.
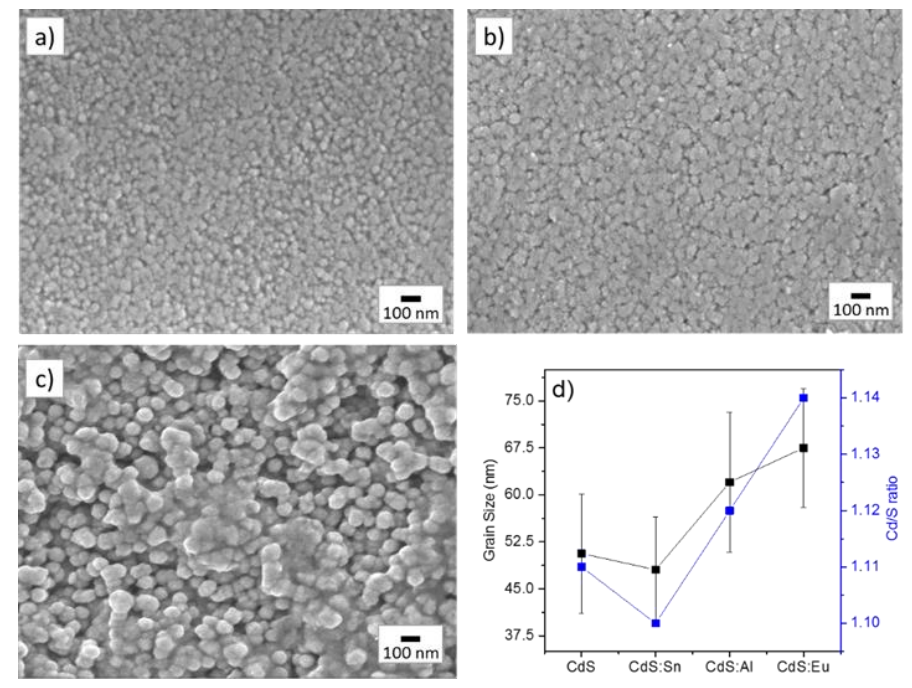

Fig. 1: SEM images for CdS thin films. Top view micrographs at 50,000 X of thin films of cadmium sulfide with different materials as dopants: a) CdS:Al, b) CdS:Sn, c) CdS:Eu and d) Grain size of metal-doped CdS thin films and relation of $\mathrm{Cd} / \mathrm{S}$.

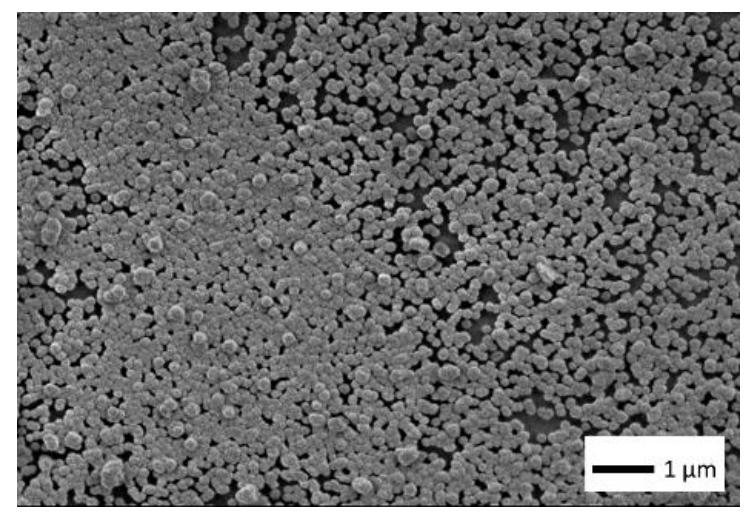

Fig. 2: SEM image for CdSe thin film.

XPS data for CdS/ITO structure (see Fig. 4 for elements distribution in CdS film of the structure) show essential deviation from average stoichiometry near the CdS film boundaries (here the abscissa gives the etching time; for example $180 \mathrm{~s}$ with etching rate of $0.66 \mathrm{~nm} / \mathrm{s}$ gives the $\mathrm{CdS}$ film thickness of $120 \mathrm{~nm}$, etching rate was calibrated with $\mathrm{Ta}_{2} \mathrm{O}_{5}$ standard). We see that CdS semiconductor film has thin (of order of $10 \mathrm{~nm}$ ) near-surface and near-interface layers with a composition different from that of the bulk. Photoluminescence (PL) results measurements (Fig. 5) reveal the blue shift of electronic transitions from the band gap energy suggesting that these near boundary layers can be treated as quantum wells with the 
corresponding quantum confinement effects. Here we see the bands at 400, 530 and $680 \mathrm{~nm}$ corresponding to photons 3.1, 2.34 and $1.83 \mathrm{eV}$. The last two are related to the defects, and according to [3] can be ascribed to $\mathrm{S}$ interstitials $(2.34 \mathrm{eV})$ and $\mathrm{I}_{\mathrm{Cd}} \mathrm{V}_{\mathrm{Cd}}$ complexes $(1.83 \mathrm{eV})$. But the first band exceeds the bulk band gap value by approximately $0.6 \mathrm{eV}$ and definitely indicates the quantum confinement effect.

The doping of CdS films has profound effect on their electrical properties enhancing the conductivity, which allowed us to prepare Schottky diodes on their basis that can be used as efficient photosensors; these results will be published separately. Here we concentrate on the discussion of quantum effects caused by nano structuration of the materials obtained.

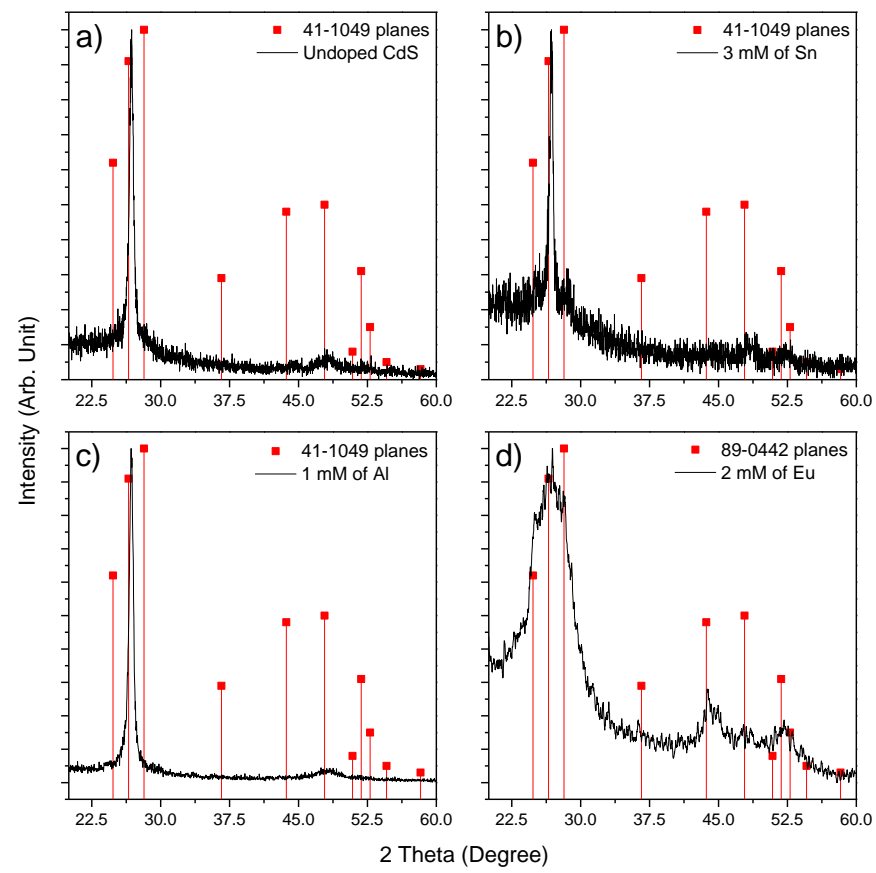

Fig. 3: XRD Diffractograms of thin films of cadmium sulfide with different materials as dopants:

a) CdS ammonia free formulation without doping, b) CdS:Al, c) CdS:Sn and d) CdS:Eu.

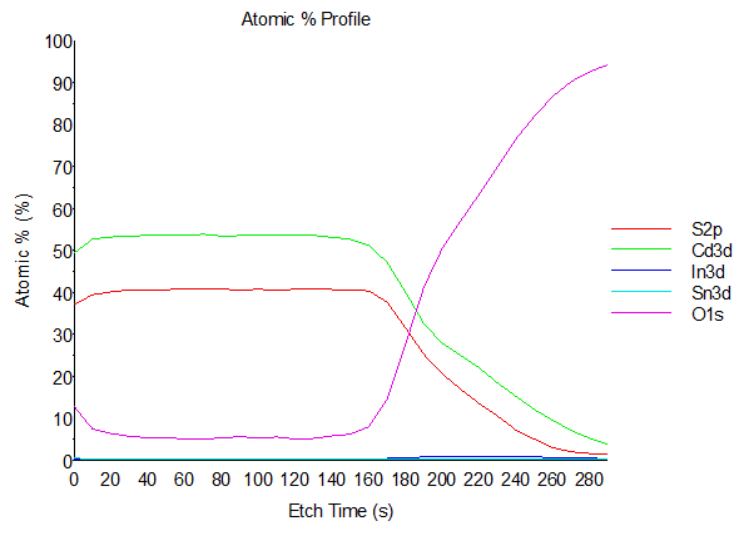

Fig. 4: Distribution of elements in sample CdS/ITO on glass.

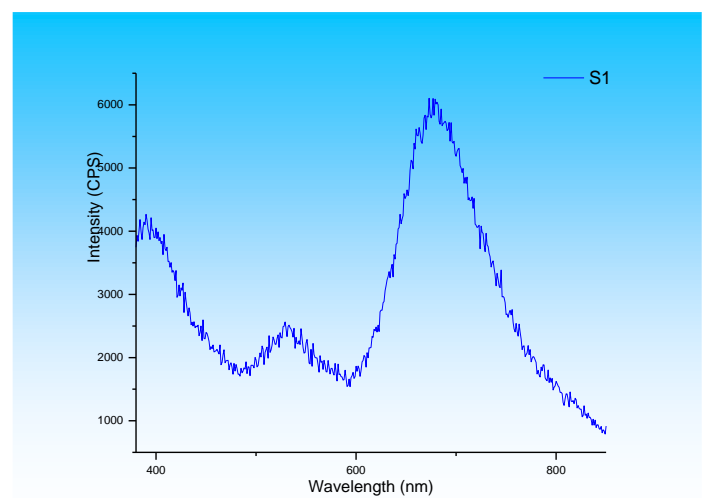

Fig. 5: Photoluminescence of CdS film. 


\subsection{Optical properties}

The results of optical study of CdS films doped with Sn (transmission and reflection spectra) are given in Fig. 6a and 6b. The upper line in Fig. $6 \mathrm{~b}$ and lower one in $6 \mathrm{a}$ give transmission and reflection of glass substrate; the samples transmission curves above $515 \mathrm{~nm}$ (photon energies less than $2.4 \mathrm{eV}$ ) show general transmission of around $70 \%$ and corresponding reflectance of around $30 \%$ indicating that the material obtained can serve as good window layer in solar cell. The average band gap value for CdS:Sn films found from optical data was equal to $2.47 \mathrm{eV}$ that is somewhat higher than the bulk value $(2.42 \mathrm{eV})$, this point will be discussed below. The data for $\mathrm{CdS}: \mathrm{Al}$ are quite similar.

Fig. 7 presents the diagram normally used for determination of the band gap for CdSe samples with the different amount of sodium citrate during preparation. It is seen that the average band gap value is $1.755 \mathrm{eV}$, a little higher than the bulk value known from the literature $(1.74 \mathrm{eV})$, the sodium nitrate amount having practically no effect on the band gap.
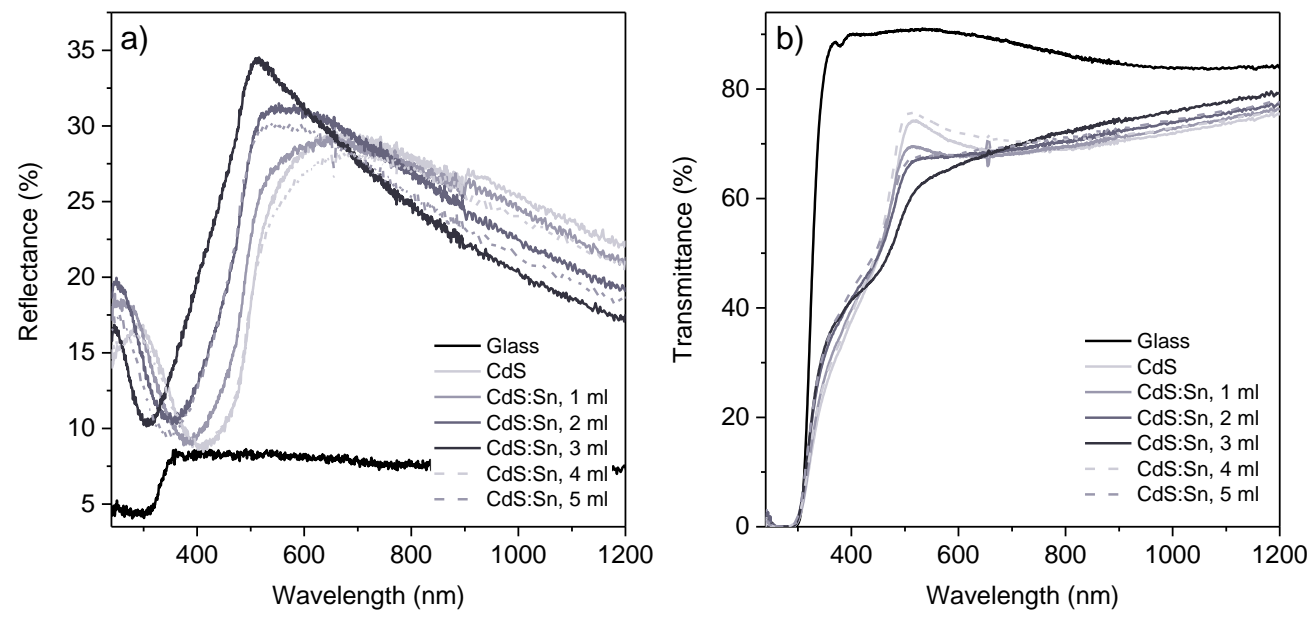

Fig. 6: Transmission and reflection spectra of CdS:Sn films.

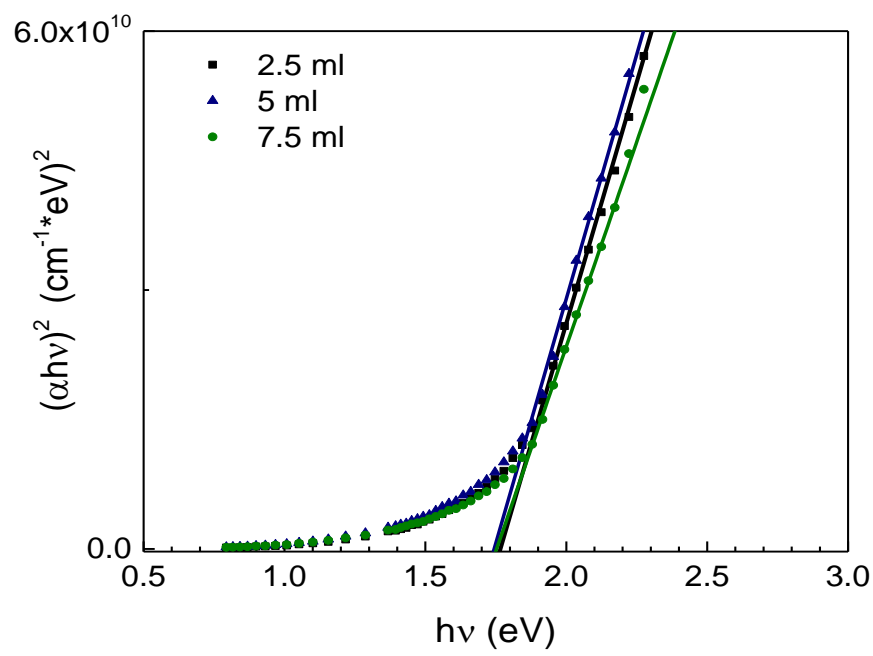

Fig. 7: CdSe films band gap from optical spectra. 


\subsection{Discussion of quantum effects}

In the materials and structures studied, two types of quantum confinement effects are observed: one connected with near-boundary quantum wells (blue shift of CdS photoluminescence spectrum), and another resulting from the material porosity (band gap blue shift found in $\mathrm{CdS}, \mathrm{CdSe}$ and $\mathrm{PbS}$ ). The first effect was analyzed in previous publication [4]; here we discuss the second one.

Our approach is based on the Even Mirror Boundary Conditions (EMBC) in solution of the Schrödinger equation (see [5-7]). Earlier we have described the porosity-stimulated effects upon the band gap of CBD-made materials [8] treating the pores as "Quantum Anti-Dots" (voids surrounded by semiconductor material, with mirror-reflecting walls allowing again application of our original Mirror Boundary Conditions). On the basis of XRD and SEM data mentioned, we assume that our samples have the closely packed structure of ball-shaped crystallites. In this case a pore is formed by four adjacent balls (see Fig. 8) and its shape can be modeled by a tetrahedral pyramid formed by planes touching the balls in centers of facets. We describe electron's interaction with the ball surface (for an electron approaching a pore from within a crystallite) equalizing its $\Psi$-function in a near-surface point with the one of its image in a spherical wall-mirror, similar to the case analyzed in [7] for a spherical quantum dot; at small distances, a spherical surface can be approximated by a plane.

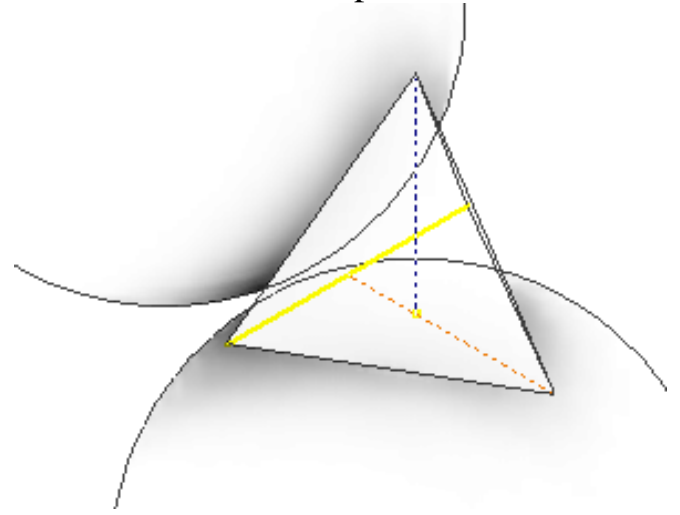

Fig. 8: Tetrahedral pyramid in the pore between closely packed balls.

In dense packing of equal spheres (theory in [9]) the spheres occupy $74 \%$ of the total volume. For every sphere there is one gap (void or pore) surrounded by six spheres (octahedral one) and two gaps surrounded by four spheres (tetrahedral). The distances to the centers of these gaps from the centers of the surrounding spheres is $\sqrt{3 / 2}$ for the tetrahedral, and $\sqrt{2}$ for the octahedral, when the sphere radius is taken as 1 . We restrict ourselves with analysis of tetrahedral pores (being smaller than octahedral ones, they cause greater quantum confinement effect).

Thus, total voids have $26 \%$ of the volume; so, their summary pore volume for one sphere is $26 / 74=0.35 \%$ of a volume of a sphere. If we neglect the octahedral void, then each tetrahedral one has $0.175 \%$ of a sphere volume. Then we approximate a tetrahedral pore as tetrahedron - a pyramid formed by 4 equilateral triangles - it gives a possibility to find a relation between tetrahedron side $a$ and diameter D (or radius $\mathrm{R}$ ) of a sphere. The volume of tetrahedron

$$
V_{t}=\frac{a^{3}}{6 \sqrt{2}}
$$

and the volume of sphere

$$
V_{s p}=\frac{\pi D^{3}}{6}
$$

from the equality $\mathrm{V}_{\mathrm{t}}=0.175 \mathrm{~V}_{\mathrm{sp}}$ we find that $a=0.92 \mathrm{D}=1.84 \mathrm{R}$. 
It is clear that this approximation gives exaggerated size of actual tetrahedral pore; we made another estimation by calculation of the volume of tetrahedron that touches four spheres in a tetrahedral pore. This approach gives $a=1.1 \mathrm{R}$. The average of these two approximations gives $a=1.47 \mathrm{R}$.

Our calculations of the energy spectra of pyramidal quantum dots based on EMBC approach (see argumentation in [10]) give for tetrahedral pyramid the expression

$$
E=0.5 \frac{\hbar^{2}}{m a^{2}} n^{2}
$$

$m$ being electron effective mass, $\hbar$ - the Planck constant, and $n$ - quantum number.

In the following discussion, we assume that typical pores in CBD materials are smaller than the Bohr radius $\alpha_{\mathrm{B}}$ for an exciton $\left(\alpha / 2<\alpha_{B}\right)$ which is important for the case of exciton formation under the illumination [11]. In all semiconductors studied $(\mathrm{CdS}, \mathrm{CdSe}, \mathrm{PbS})$ the value of $\alpha_{\mathrm{B}}$ exceeds $20 \mathrm{~nm}$ according to the expression below

$$
\alpha_{B}=\frac{4 \pi \hbar^{2} \epsilon \epsilon_{0}}{\mu e^{2}}
$$

with exciton reduced mass

$$
\mu=\frac{m_{e} m_{h}}{\left(m_{e}+m_{h}\right)}
$$

Here, $\mathrm{m}_{\mathrm{e}, \mathrm{h}}$ is the electron/hole effective mass, $\epsilon$ is the dielectric constant of the material, and $\epsilon_{\mathrm{o}}$ is a permittivity constant. Following the argumentation given in $[5,11]$, we see that for the blue shift estimation one can directly apply the expressions for energy spectra because the separation between the quantum levels proportional to $\hbar^{2} / m a^{2}$ is large compared to the Coulomb interaction between the carriers which is proportional to $e^{2} / \epsilon \epsilon_{o} \alpha$. Therefore, Coulomb interaction can be neglected, and the energy levels could be found from quantum confinement effect alone. Accordingly, we shall calculate the emission/absorption photon energy for transitions corresponding to the exciton ground state, which is given in (3) by $n=1$. Thus, we will assume that the fundamental absorption edge corresponds to generation of an exciton with ground state energy:

$$
\hbar v_{\min }=E_{g}+\frac{\hbar^{2}}{2 \mu a^{2}}
$$

$\mathrm{E}_{\mathrm{g}}$ being the semiconductor bulk band gap.

The bulk $\mathrm{E}_{\mathrm{g}}$ value in $\mathrm{CdS}$ at room temperature is $2.42 \mathrm{eV}$. Our optical data give band gap value of $2.47 \mathrm{eV}$ for $\mathrm{Sn}-\mathrm{doped}$ thin films prepared. To explain the edge shift $h v_{\min }-E_{g}$ of $0.05 \mathrm{eV}$ observed (exciton reduced mass [12] $0.134 \mathrm{~m}_{0}$ ), one obtains from (6) the tetrahedral pore size $a=13 \mathrm{~nm}$. Taking the relation above between the value of $a$ and the "ball radius"

$\mathrm{R}$ as $a / R=1.47$, we obtain the ball radius $\mathrm{R}$ equal to $19 \mathrm{~nm}$ that quite agrees with the crystallite size cited above. Thus, we see that our assumption about the internal porosity as a cause of band gap blue shift agrees with the experimental data.

\section{Conclusion}

Two kinds of nano-structuration were observed and investigated in chalcogenide films prepared for application in photovoltaic converters, both causing the quantum confinement effects and the corresponding blue shift of electronic transitions: one due to variation of films composition near their boundaries, and another due to porosity inherent to all materials produced by Chemical Bath Deposition (thus we conclude that used in our work Light Assisted Chemical Bath Deposition - LACBD also possess this quality). Theoretical modeling of pore-induced blue shift based on our original Mirror 
Boundary Conditions in "particle in a box" quantum problem agrees with experimental data. All effects examined can be used for monitoring of materials and structures parameters and are profitable for photovoltaic converters.

\section{Acknowledgements}

F. Willars-Rodriguez and F. Chimal-Moreno wish to thank CONACYT for their scholarship. We are thankful to Prof. Paul Horley for fruitful discussion of quantum effects, and want to acknowledge the technical assistance of: Luis Gerardo Silva Vidaurri, C.A. Avila Herrera, J.E. Urbina Alvárez, A. Jiménez Nieto.

\section{References}

[1] J. Hernandez Borja, Y. V. Vorobiev, R. Ramirez Bon, "Thin film solar cells of CdS/PbS chemically deposited by an ammonia-free process" Sol. En. Mat. Solar Cells, vol. 95, pp. 1882-1888, 2011.

[2] H. E. Esparza-Ponce, J. Hernández-Borja, A. Reyes-Rojas, M. Cervantes-Sánchez, "Growth technology, X-ray and optical properties of CdSe thin films" Mater. Chem. Phys., vol. 113, pp. 824-828, 2009.

[3] D. Wu et al. "Self-powered and fast-speed photodetectors based on CdS:Ga nanoribbon/Au Schottky diodes" J. Mater. Chem. vol. 22, pp. 23272-23276, 2012.

[4] Y. V. Vorobiev, I. R. Chávez-Urbiola, S. A. Pérez-García, P. Vorobiev, "Quantum confinement effects in a GLASS/ITO/CdS/CdTe superstrate structure designed for solar energy conversion", Proceedings of ISER International Conference, Paris, France, 11th-12th October 2016, ISBN: 978-93-86083-34-0.

[5] Y. V. Vorobiev, P. M. Gorley, V. R. Vieira, P. P. Horley, J. González-Hernández, T. V. Torchynska, A. Diaz Cano, "Effect of Boundary Conditions on the Energy Spectra of Semiconductor Quantum Dots Calculated in the Effective Mass Approximation", Physica E, vol. 42, pp 2264-2267, 2010

[6] Y. V. Vorobiev, T. V. Torchynska, P. P. Horley, "Effect of Aspect Ratio on Energy of Optical Transitions in a PyramidShaped Quantum Dot", Physica E, vol. 51, pp 42-47, 2013

[7] Y. V. Vorobiev, P. M Gorley, V. R. Vieira and P. P. Horley, "Electronic States in Nanostructures and Quantum Dots", Proceedings of International Conference on Innovative Technologies (Bahadurgarh, India, 2009), pp. 19-29.

[8] Y. V. Vorobiev, P. P. Horley, J. Hernandez-Borja, H. E. Esparza-Ponce, R. Ramirez-Bon, P. Vorobiev, C. Perez and J. Gonzalez-Hernandez, "The effects of porosity on optical properties of semiconductor chalcogenide films obtained by the chemical bath deposition", Nanoscale Research Letters, vol. 7, 483, 2012

[9] J. H. Conway, N. J. Sloane, A. B. Eiichi, in Sphere packings, lattices, and groups. Springer. 1999, Section 6.3.

[10] F. J. Willars-Rodríguez, I. R. Chávez-Urbiola, M. A. Hernández-Landaverde, P. Vorobiev, R. Ramirez-Bon, Y. V. Vorobiev, "Effects of tin-doping on cadmium sulfide $(\mathrm{CdS}: \mathrm{Sn})$ thin-films grown by light-assisted chemical bath deposition process for solar photovoltaic cell", Thin Solid Films, vol. 653 (2018), pp. 341-349

[11] S. V. Gaponenko, Optical Properties of Semiconductor Nanocrystals. Cambridge: Cambridge University Press; 1998.

[12] J. Singh, Physics of Semiconductors and Their Heterostructures. New York: McGraw-Hill, 1993. 